\title{
Optical-resolution confocal photoacoustic microscopy
}

Konstantin Maslov, Hao F. Zhang, Song Hu, Lihong V. Wang

Konstantin Maslov, Hao F. Zhang, Song Hu, Lihong V. Wang, "Opticalresolution confocal photoacoustic microscopy," Proc. SPIE 6856, Photons Plus Ultrasound: Imaging and Sensing 2008: The Ninth Conference on Biomedical Thermoacoustics, Optoacoustics, and Acousto-optics, 68561I (28 February 2008); doi: 10.1117/12.763889

SPIE. Event: SPIE BiOS, 2008, San Jose, California, United States 


\title{
Optical-Resolution Confocal Photoacoustic Microscopy
}

\author{
Konstantin Maslov ${ }^{\mathrm{a}}$, Hao F. Zhang ${ }^{\mathrm{a}, \mathrm{b}}$, Song Hu $\mathrm{u}^{\mathrm{a}}$, Lihong V. Wang ${ }^{\mathrm{a}}$ \\ ${ }^{a}$ Optical Imaging Laboratory, Department of Biomedical Engineering, \\ Washington University in St. Louis, St. Louis, Missouri 63130 \\ ${ }^{\mathrm{b}}$ Current address: Department of Electrical Engineering and Computer Science, \\ University of Wisconsin-Milwaukee, P.O. Box 748 Milwaukee, WI 53201-0784
}

\begin{abstract}
A confocal photoacoustic microscope with improved lateral resolution has been developed by employing stronglyfocused bright field optical illumination and spherically focused $75-\mathrm{MHz}$ ultrasonic detection. The lateral resolution was experimentally measured to be $5 \mu \mathrm{m}$ and the axial resolution was estimated to be $15 \mu \mathrm{m}$. The maximum imaging depth was demonstrated to be greater than $0.7 \mathrm{~mm}$. In in vivo experiments, microvessels with a diameter of $\sim 5 \mu \mathrm{m}$ are imaged in small animals.
\end{abstract}

Keywords: photoacoustic tomography, confocal microscopy

\section{INTRODUCTION}

Capillaries, the smallest blood vessels, are the distal end of the vasculature system where oxygen and nutrients are exchanged between blood and tissue. Hence, noninvasive imaging of capillaries in vivo has long been desired as a window to studying fundamental physiological phenomena, such as neurovascular coupling ${ }^{1,2}$. Existing imaging modalities, however, cannot provide the required sensitivity, contrast, and spatial resolution simultaneously ${ }^{3}$.

Spatial resolution of almost all existing photoacoustic (PA) imaging devices depends on ultrasonic parameters. For example, in dark-field photoacoustic microscopy (PAM), the lateral resolution is determined by the focusing ability of ultrasonic transducer, which depends on the ultrasonic transducer center frequency and numerical aperture (NA). A lateral resolution of $50 \mu \mathrm{m}$ has been achieved with a center frequency of $50 \mathrm{MHz}$ and a NA of $0.44^{4-6}$. This lateral resolution is adequate for many biomedical applications. However, to resolve smaller structures such as capillaries (3-7 $\mu \mathrm{m}$ in diameter ${ }^{7,8}$ ) with optical-absorption contrast, further improvement of the spatial resolution is required. Of course, this improvement has to be achieved at the expense of maximum imaging depth.

If such an improvement is provided by increasing the ultrasonic focusing capability, a $5-\mu \mathrm{m}$ lateral resolution will require an ultrasonic center frequency higher than $300 \mathrm{MHz}$. At this frequency ultrasonic attenuation exceeds $20 \mathrm{~dB} / \mathrm{mm}$ even in immersion liquid (water), which limits the penetration depth of acoustic wave in tissue to $\sim 0.1 \mathrm{~mm}$. An alternative and, perhaps, easier way to improve the lateral resolution is to apply fine optical focusing. The optical transport mean free path in biological tissue is $1 \mathrm{~mm}$, which limits the maximum imaging depth to comparable value of $\sim 1 \mathrm{~mm}$. The advantages of using fine optical focus include: (1) less laser pulse energy is required to reach the same optical fluence in image plane compared with the previous realizations; (2) better focusing capability because due to nonlinear nature of photoacoustic effect acoustic wave amplitude is proportional to light intensity (square of amplitude). Some investigators had explored this approach before with different emphases, such as flow cytometry and different detection technique ${ }^{9,10}$.

In this paper, we report on the reflection-mode confocal PAM system that employs a strong optical focusing with a bright field optical illumination. The optical focal diameter is several times smaller than the ultrasonic focal diameter. The maximum imaging depth and lateral resolution are primarily limited by the optical scattering in tissue; the axial resolution is determined by the ultrasonic bandwidth.

Photons Plus Ultrasound: Imaging and Sensing 2008: The Ninth Conference on Biomedical

Thermoacoustics, Optoacoustics, and Acousto-optics, edited by Alexander A. Oraevsky, Lihong V. Wang,

Proc. of SPIE Vol. 6856, 68561I, (2008) - 1605-7422/08/\$18 - doi: 10.1117/12.763889

Proc. of SPIE Vol. 6856 68561I-1 


\section{METHODS AND MATERIALS}

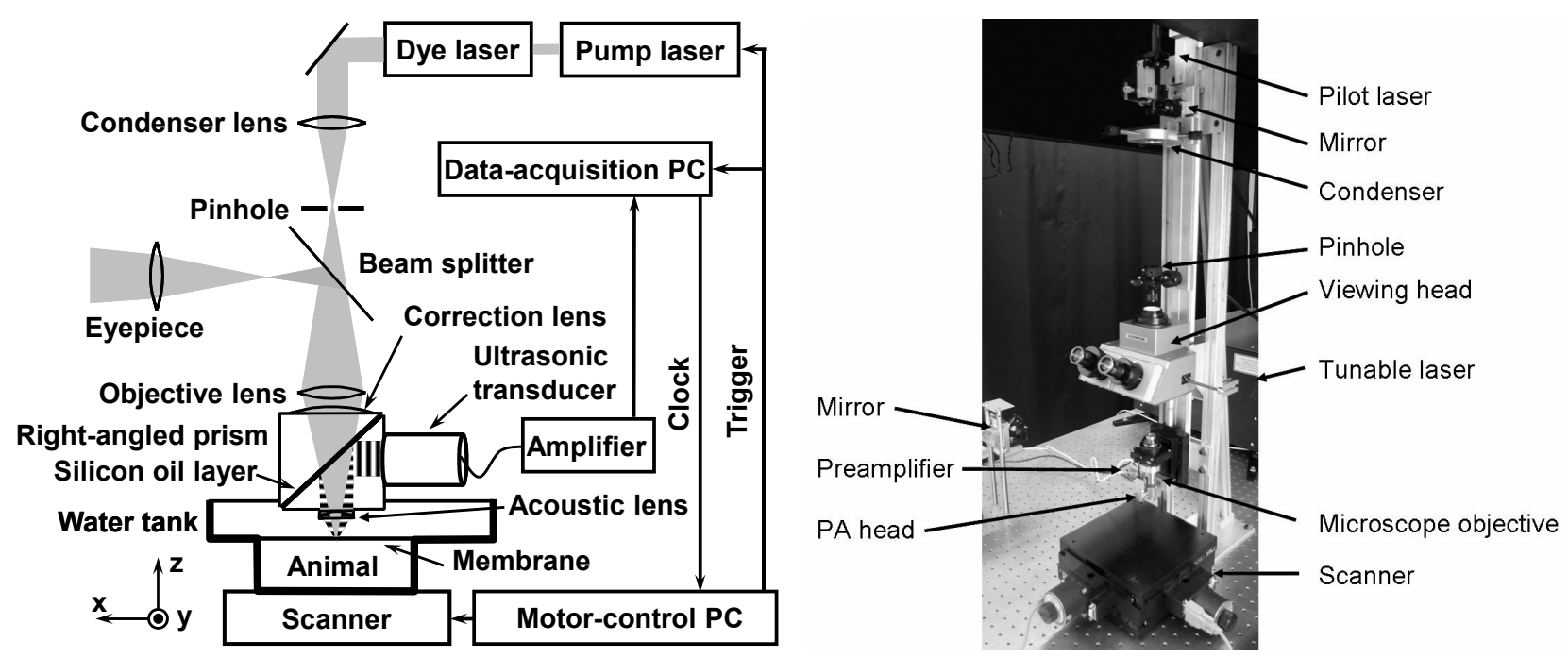

Fig. 1. Experimental setup.

The schematic of the experimental setup is shown in Fig. 1. A Nd:YLF laser (INNOSLAB, Edgewave) pumped dye laser (CBR-D, Sirah) was used as the irradiation source. The laser pulse duration is $5 \mathrm{~ns}$ and the pulse repetition rate, which is controlled by the external trigger signal, was as high as $2 \mathrm{kHz}$. The light coming out of the dye laser passed through a spatial filter (25- $\mu \mathrm{m}$ pinhole, P250S, Thorlabs) and was then focused by a microscope objective lens (RMS4X, Thorlabs; NA: 0.1; focal length: $45 \mathrm{~mm}$; working distance: $22 \mathrm{~mm}$ ). The distance between the pinhole and the objective lens is 400 $\mathrm{mm}$, which gives diffraction limited optical focusing of $3.7 \mu \mathrm{m}$ in diameter and a focal zone of $200 \mu \mathrm{m}$ in water. The laser pulse energy after the objective lens was measured to be $100 \mathrm{~nJ}$. An optional beam splitter was located between the pinhole and the objective lens to facilitate the manual adjustment and system alignment. Two right angle prisms (NT32545 , Edmund Optics) were placed together on their hypotenuses to form a cube with a gap of $0.1 \mathrm{~mm}$ in between. The gap was filled with $1000 \mathrm{cSt}$ silicon oil. The silicon oil and the glass had good optical refractive index match (glass: 1.5; silicon oil: 1.4) but large acoustic impedance mismatch (glass: $12.1 \times 10^{6} \mathrm{~N} \cdot \mathrm{s} / \mathrm{m}^{3}$; silicon oil: $0.95 \times 10^{6} \mathrm{~N} \cdot \mathrm{s} / \mathrm{m}^{3}$ ). As a result, this silicon oil layer is optically transparent but acts as an acoustic reflector. An ultrasonic transducer (V2012-BC, Panametrics-NDT) with a center frequency of $75 \mathrm{MHz}$, a $3 \mathrm{~dB}$ bandwidth of $80 \%$, and an active element diameter of $6.35 \mathrm{~mm}$ was attached to a cathetus of the bottom prism as shown in Fig. 1. An acoustic lens (geometrical radius of curvature: $5.2 \mathrm{~mm}$; diameter: $6.35 \mathrm{~mm}$ ) was attached to the bottom of the cube. This acoustic lens has a NA of 0.46 in water and gives an ultrasonic focal spot size of $27 \mu \mathrm{m}$.

The PA signals detected by the ultrasonic transducer were amplified by $48 \mathrm{~dB}$ (ZFL 500LN, Mini-Circuits) before being digitized by a digital acquisition board (CompuScope 12400, Gage Applied). A motor driving PC controlled the scanner to perform raster scanning and sent trigger signals to both the data-acquisition PC and the pump laser. The trigger signals were synchronized with the clock-out signals from the digital acquisition board to minimize jitter. A more detailed description of the trigger sequence can be found elsewhere ${ }^{11}$.

A window was opened at the bottom of the water tank and was sealed with an ultrasonically and optically transparent polyethylene membrane (thickness: $25 \mu \mathrm{m}$ ). Animal was placed under the water tank with the region of interest (ROI) exposed directly below the window. Ultrasonic gel (Clear Image, SonoTech) was applied to the ROI to achieve good acoustic coupling. Before data acquisition, the water tank was filled with de-ionized water and the acoustic lens, as well as the bottom of the prism cube, was immersed in water. Here, the raster scanning was implemented by moving the sample instead of the optical-acoustic dual-foci for simplicity. During data acquisition, the water tank and the animal were translated together along the horizontal $(x-y)$ plane. Same as the previous PAM system ${ }^{5}$, a volumetric image was acquired by combining time-resolved recording of PA signal at each horizontal location with a two-dimensional (2D) raster scanning. One-dimensional (1D) PA signal (A-line) was recorded for $2 \mu$ s at a sampling rate of $200 \mathrm{MS} / \mathrm{s}$. Cross- 
sectional (B-scan) image and maximum amplitude projection (MAP) images were used to visualize the acquired volumetric PA data.

The in vivo experiments were carried out to image microvasculature in a nude mouse (Hsd:Athymic Nude-Foxn $1^{\mathrm{NU}}$, Harlan Co.; body weight: $20 \mathrm{~g}$ ) ear at the optical wavelength of $570 \mathrm{~nm}$. Nude mouse ear model (thickness: around 300 $\mu \mathrm{m})$ has well-developed vasculature and has been widely used to study tumor angiogenesis and other microvascular diseases $^{12,13}$.

During image acquisition, animal was kept motionless using a breathing anesthesia system (E-Z Anesthesia, Euthanex) and was kept warm by an infrared lamp. Unlike previous studies ${ }^{9}$, no optical clearing agent was applied to the skin surface. An area of $1 \mathrm{~mm}^{2}$ was scanned at a scanning step size of $1.25 \mu \mathrm{m}$. The scanning speed was up to 2000 steps per second. The scanning time for a complete volumetric dataset was $\sim 15 \mathrm{~min}$. After experiment, the animal recovered naturally without observable laser damage. All experimental animal procedures were carried out in conformity with the laboratory animal protocol approved by the School of Medicine Animal Studies Committee of Washington University in St. Louis.

\section{EXPERIMENTS IN VITRO}

\subsection{Resolution test}

The lateral resolution of this bright-field confocal PAM system was experimental measured by imaging an Air Force resolution test target (USAF-1951, Edmund) and a 6- $\mu \mathrm{m}$ carbon fiber immersed in water (Fig. 2). Images were acquired at the optical wavelength of $590 \mathrm{~nm}$ and no signal averaging was performed during data acquisition. In Fig. 2a and 2b, the highlighted well-resolved bars have the separation of $9.8 \mu \mathrm{m}$ (group 6, element 5). The lateral resolution was estimated to be about $5 \mu \mathrm{m}$ from extrapolation of the modulation transfer function extracted from Fig. 2a to its cutoff spatial frequency. In Fig. 2c, the mean full-width-half-max (FWHM) value of the imaged fiber is $9.8 \mu \mathrm{m}$. Hence, the lateral resolution should lie between 3.8-5 $\mu \mathrm{m}$, which agrees with the estimation of the size of Airy disk. The axial resolution was estimated to be around $15 \mu \mathrm{m}$ based on the measured $6 \mathrm{~dB}$ transducer bandwidth $(\sim 100 \mathrm{MHz})$ and the speed of sound in tissue. Both the lateral and the axial resolutions decrease with imaging depth due to increasing optical scattering and wavelength-dependent acoustic attenuation in tissue, respectively.

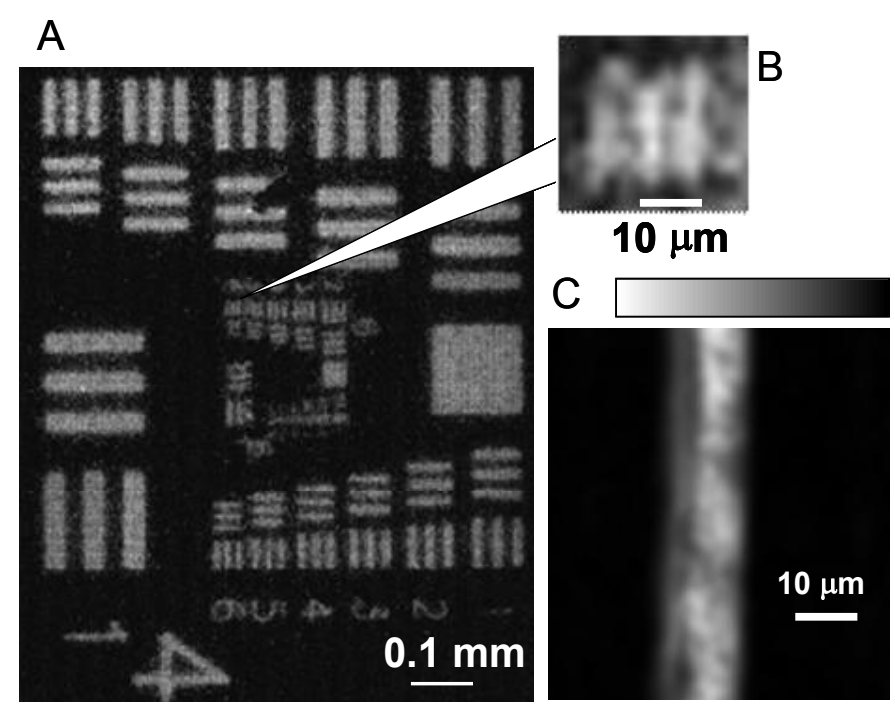

Fig. 2. Photoacoustic MAP images of an USAF-1951 resolution test target (A, B) and $6 \mu$ m diameter carbon fiber (C).

\subsection{Maximum imaging depth}

The maximum imaging depth of this system depends on the optical transport mean free path in tissue. It was measured by imaging a phantom made by two horse hairs (diameter: $\sim 200 \mu \mathrm{m}$ ) and a rat scalp piece harvested immediate after a rat was sacrificed (Fig. 3). The two horse hairs were respectively placed on top of and below the skin piece. Image was 
acquired at the optical wavelength of $630 \mathrm{~nm}$. To estimate the potential maximum imaging depth, we measured the distance between the bottom of the top hair and the top of the bottom hair. From the B-scan image shown in Fig. 3 (32 times signal averaging for each A-line), the distance was $700 \mu \mathrm{m}$. As a result, depending on the employed optical wavelength, this confocal PAM system shows a potential maximum imaging depth comparable with optical coherence tomography.

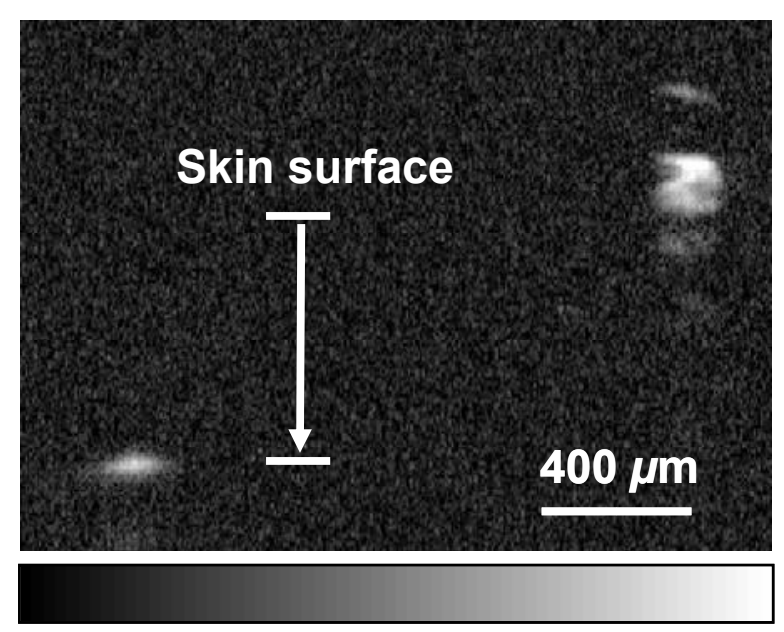

Fig. 3. The maximum imaging depth of the OR-PAM system.

\subsection{Imaging of cells in vitro.}
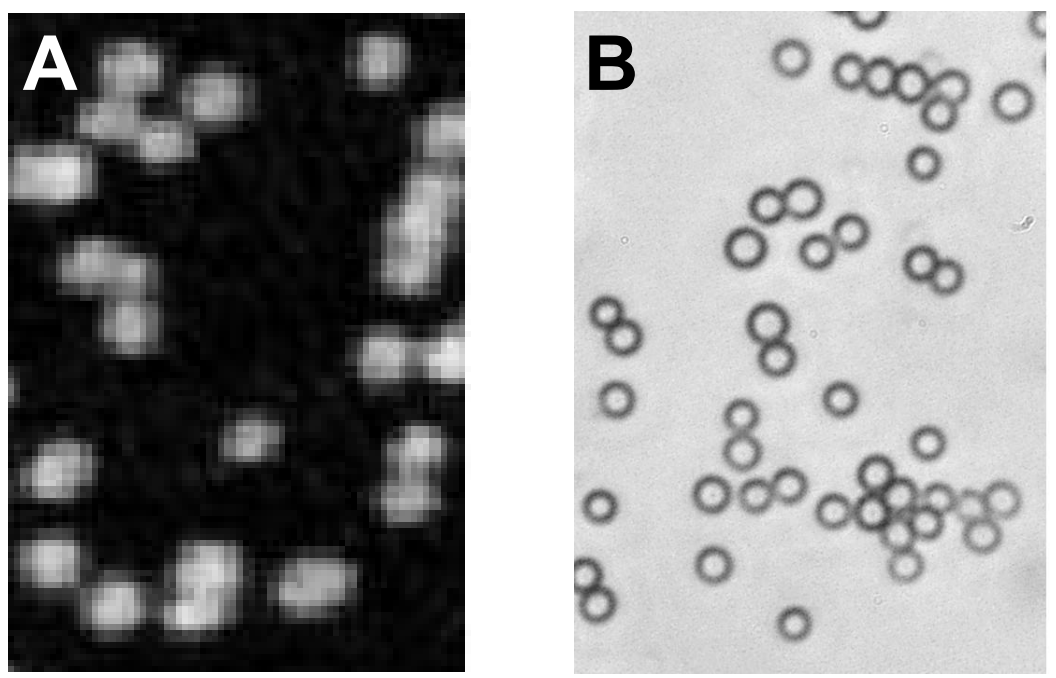

Fig. 4. MAP image of the bovine red blood cells in vitro. (A) Photoacoustic image; (B) optical microscope image.

Figure 4A shows the OR-PAM image of a smear of defibrinated bovine blood (Materials Bio, Inc), where individual RBCs are clearly resolved with high $(\sim 30 \mathrm{~dB})$ contrast. For comparison, a transmission optical microscopic image of a bovine blood sample with a similar RBC density is given in Fig. 4B. Therefore, OR-PAM has enough contrast and resolution to image single RBCs. Monitoring the motion and $\mathrm{sO}_{2}$ of $\mathrm{RBCs}$ in capillaries together can be potentially used to estimate the microscopic metabolic rate of oxygen consumption. 


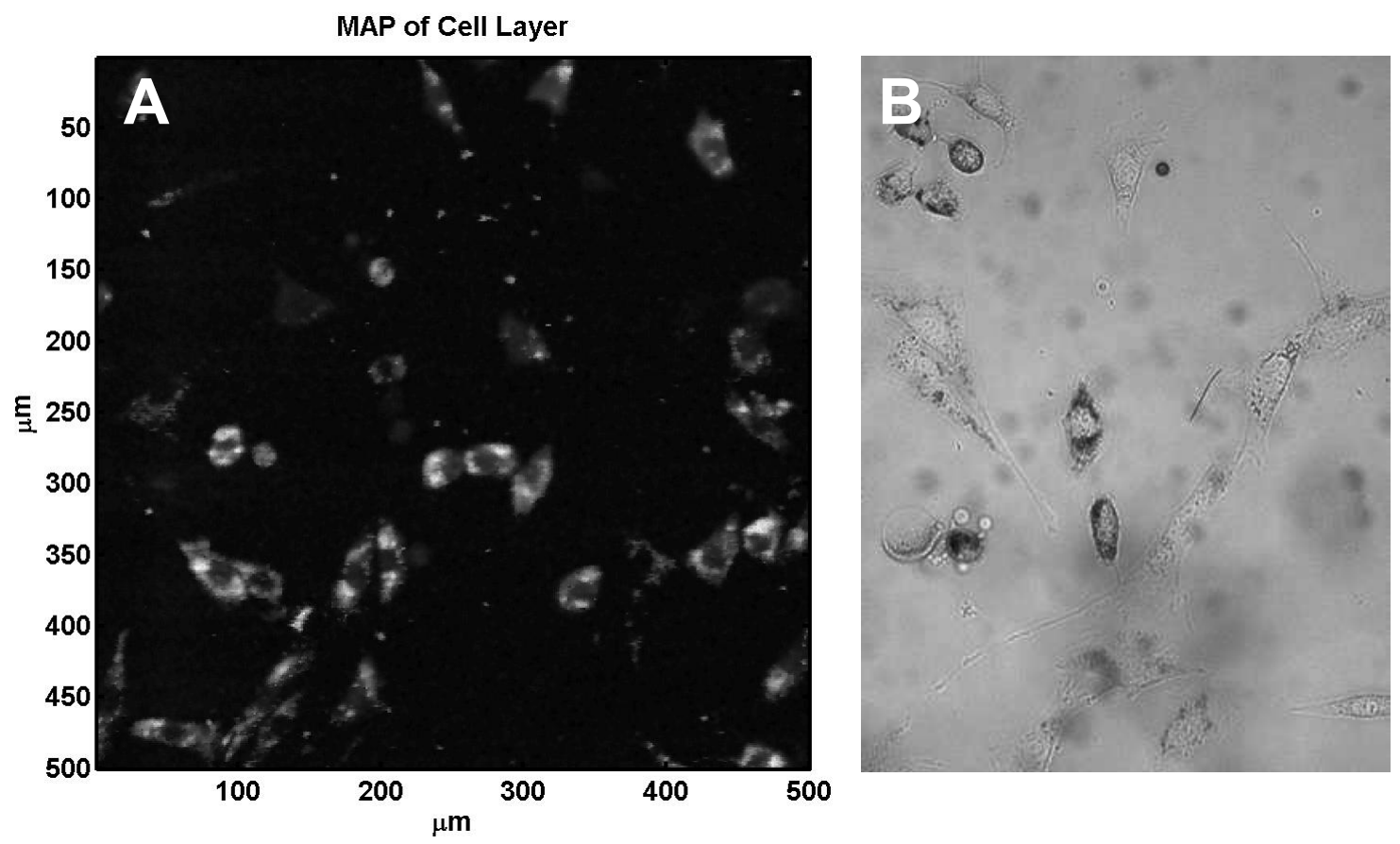

Fig. 5. MAP image of the 3T3 fibroblast culture cells in vitro. (A) Photoacoustic image; (B) optical microscope image.

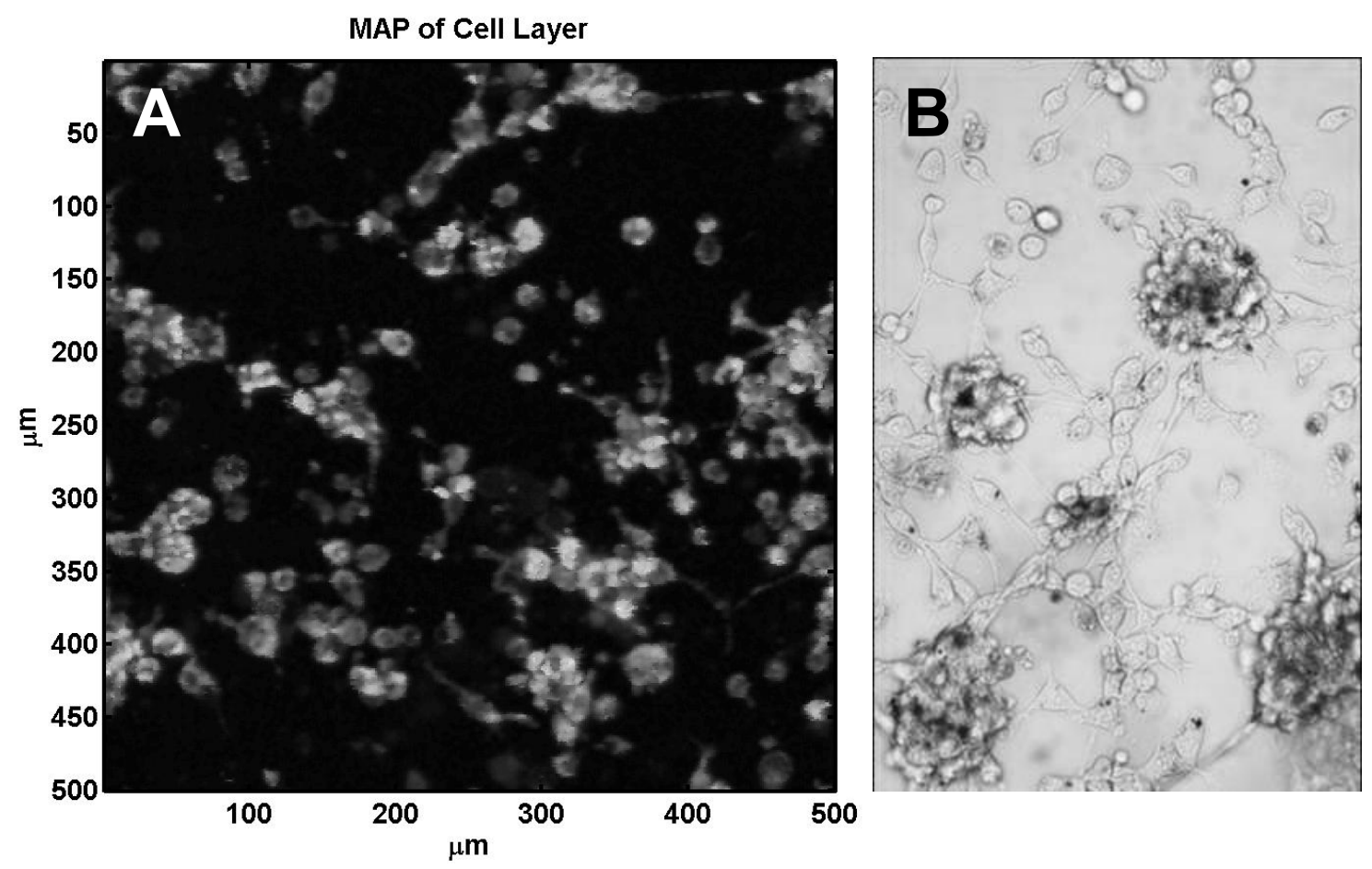

Fig. 6. MAP image of the Ct26.wt carcinoma culture cells in vitro. (A) Photoacoustic image; (B) optical microscope image.

Imaging of tissue cells, unlike RBCs, imposes a significant challenge due to their low optical absorption. Cell nuclei and cytoplasm of living cells are practically invisible to PAM. Fibroblast and carcinoma culture cells in Petri dish can be seen by OR-PAM with $2 \mathrm{M}$ methylene blue staining (figure 5 and 6, respectively). However, for both fibroblasts and carcinoma cells, the endogenetic optical absorption is very small compared with that of RBC, which guarantees high imaging contrast of blood vessels. 


\section{NONINVASIVE IMAGING IN VIVO}
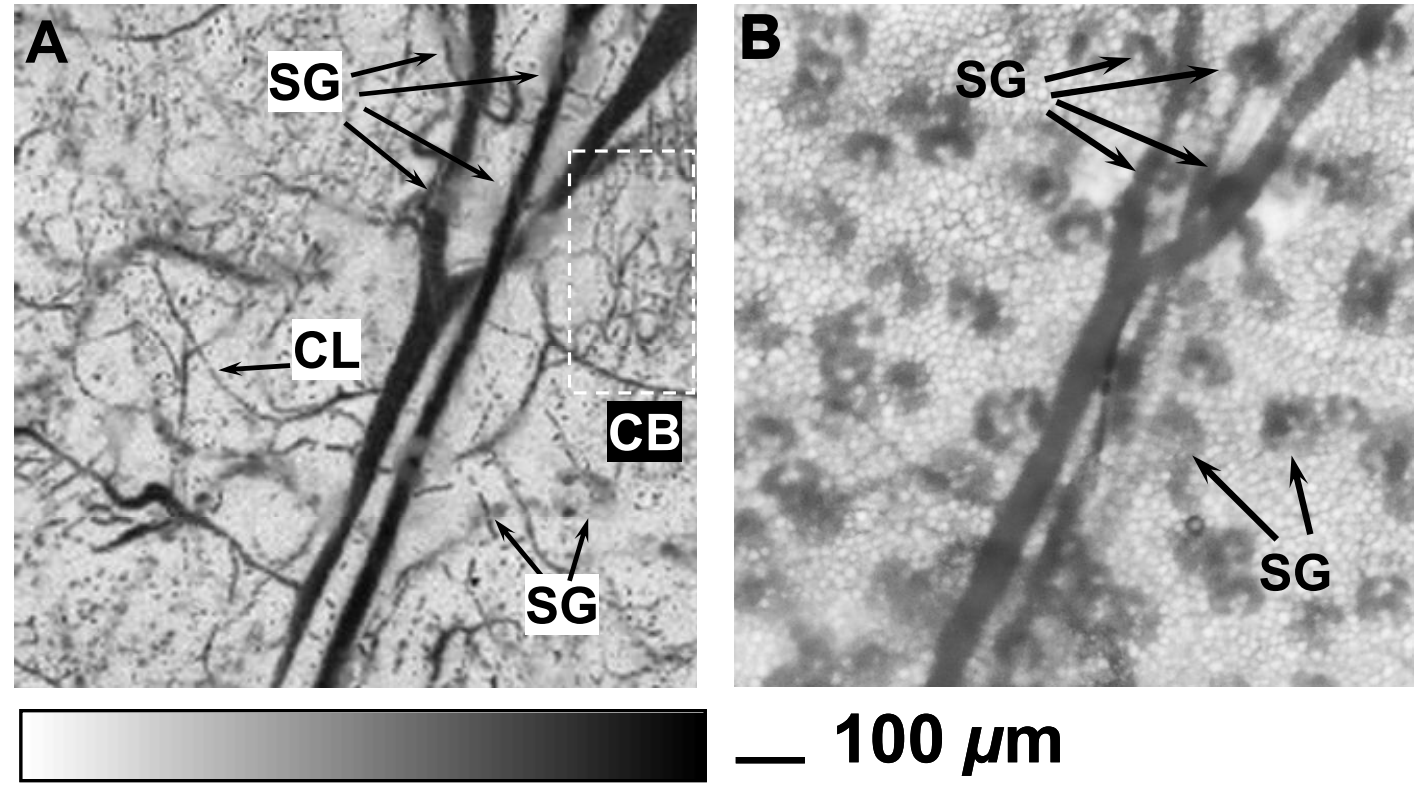

\section{$100 \mu \mathrm{m}$}

Fig. 7. Microvasculature in a nude mouse ear. (A) In vivo PA image; (B) Photograph using trans-illumination. (SG) sebaceous glands, (CL) capillaries, (CB) capillary bed.

The MAP image of the microvasculature (Fig. 7A) acquired by this confocal PAM system agrees with the photo (Fig. 7B) taken from a transmission microscopy with a $4 \times$ magnification. The mean ratio of the PA amplitudes between the blood vessels and the background is $40 \mathrm{~dB}$, which demonstrates a high optical-absorption-based imaging contrast. As highlighted in Fig. 7A, the shadows are caused by light scattering from the sebaceous glands (also in Fig. 7B). Some vessels, e.g. the one highlighted by CL in Fig. 7A, only occupies a single resolvable pixel, which presumably indicates a capillary or a metarteriole with a diameter of $\sim 5 \mu \mathrm{m}$. Parallel-going arteriole-venule pair and their further branching are also clearly observed in both the PA image and the photograph.
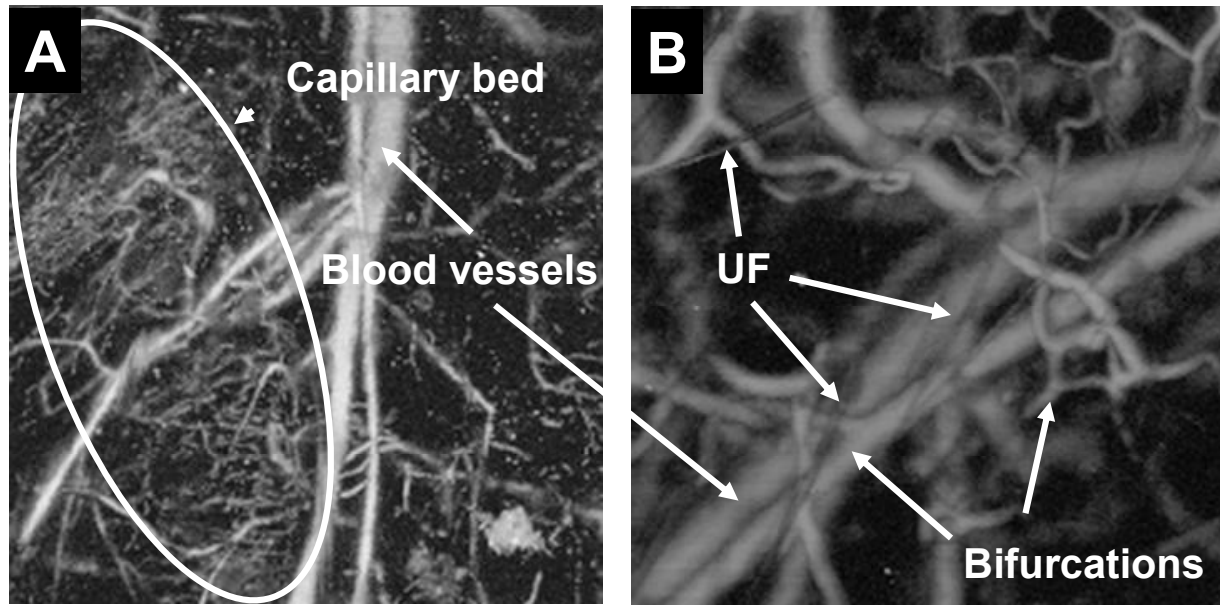

\section{$200 \mu \mathrm{m}$}

Fig. 8. In vivo OR-PAM images of an ear of a Swiss Webster mouse. (A) Peripheral area. (B) Pinna central area $4 \mathrm{~mm}$ from the ear channel. Depths of optical focusing: $30 \mu \mathrm{m}$ for (A) and $100 \mu \mathrm{m}$ for (B). (UF) unidentified fibers.

Figure 8 shows additional examples of OR-PAM images of capillary beds and blood vessel bifurcations. In addition, unidentified fibers are observed to wrap around blood vessels. This experiment further demonstrates the potential broad applications of OR-PAM. 


\section{CONCLUSION}

A bright-field confocal PAM system with an optical resolution has been reported. The lateral and axial resolutions were $5 \mu \mathrm{m}$ and $15 \mu \mathrm{m}$, respectively. The imaging depth was experimentally quantified to be greater than $0.7 \mathrm{~mm}$. Micro vessels down to $5-\mu \mathrm{m}$ - diameter are imaged in small animals in vivo.

It should be noted that the reported images represent preliminary results rather than best possible one. Current design has been chosen for its simplicity. Several technical improvements are desired in the future. Better design should increase signal amplitude for more than $10 \mathrm{~dB}$. Additional SNR improvement can be achieved by using ultrasonic lens with acoustic antireflection coating.

\section{ACKNOWLEDGEMENT}

This project is sponsored in part by National Institutes of Health Grant Nos. R01 CA106728 and R01 NS46214 (BRP).

\section{REFERENCES}

[1] B. M. Ances, "Coupling of changes in cerebral blood flow with neural activity: what must initially dip must come back up," Journal of Cerebral Blood Flow \& Metabolism 24, 1-6 (2003).

[2] H. Girouard, C. Iadecola, "Neurovascular coupling in the normal brain and in hypertension, stroke, and Alzheimer disease," Journal of Applied Physiology 100, 328-335 (2006).

[3] D. M. McDonald, P. L. Choyke, "Imaging of angiogenesis: from microscopic to clinic," Nature Medicine 9, 713725 (2003).

[4] K Maslov, G. Stoica, and L. V. Wang, "In vivo dark-field reflection-mode photoacoustic microscopy," Optics Letters 30, 625-627 (2005).

[5] H. F. Zhang, K. Maslov, G. Stoica, and L. V. Wang, "Functional photoacoustic microscopy for high-resolution and noninvasive in vivo imaging," Nature Biotechnology 24, 848-851 (2006).

[6] H. F. Zhang, K, Maslov, and L. V. Wang, "In vivo imaging of subcutaneous structures using functional photoacoustic microscopy," Nature Protocols 4, 797-804 (2007).

[7] http://en.wikipedia.org/wiki/Capillary.

[8] R. F. Potter, A. C. Groom, "Capillary diameter and geometry in cardiac and skeletal muscle studied by means of corrosion casts," Microvascular Research 25, 68-84 (1983).

[9] V. P. Zharov, E. I. Galanzha, E. V. Shashkov, N. G. Khlebtsov, and V. V. Tuchin, "In vivo photoacoustic flow cytometry for monitor of circulating single cancer cells and contrast agents," Optics Letters 31, 3623-3625 (2006).

[10]E. Savateeva, A. Karabutov, M. Motamedi,B. Bell,J. Brent;R. Johnigan, and A. Oraevsky, "Noninvasive detection and staging of oral cancer in vivo with confocal opto-acoustic tomography," Proceedings of SPIE 3916, 55-66 (2000).

[11] K. Maslov, H. F. Zhang, and L. V. Wang, "Portable real-time photoacoustic microscopy," Proceedings of the SPIE 6437, 643727 (2007).

[12] S. Basu, J. A. Nagy, S. Pal, E. Vasile, I. A. Eckelhoefer, V. S. Bliss, E. J. Manseau, P. S. Dasgupta, H. F. Dovrak, and D. Mukhopadhyay, "The neurotransmitter dopamine inhibits angiogenesis induced by vascular permeability factor/vascular endothelial growth factor," Nature Medicine 7, 569-574 (2001).

[13]D. A. Elson, G. Thurston, L. E. Huang, D. G. Ginzinger, D. M. McDonald, R. S. Johnson, and J. M. Arbeit, "Induction of hypervascularity without leakage or inflammation in transgenic mice overexpressing hypoxiainducible factor-1 $\alpha, "$ Genes \& Development 15, 2520-2532 (2001). 